\title{
122. Combined application of low intensity pulsed ultrasound in dental implantation
}

\author{
A. Bubulis ${ }^{1}$, S. Rubnikovich ${ }^{2}$, I. Khomich ${ }^{3}$, Y. Denisova ${ }^{4}$, Vl. Minchenya ${ }^{5}$ \\ ${ }^{1}$ Kaunas University of Technology, Institute of Mechatronics, Studentu str. 56, 51424 Kaunas, Lithuania \\ ${ }^{2,3}$ Belarusian Medical Academy of Postgraduate Education Republic of Belarus, \\ Brovki d. 3-3, Minsk, 220013, Republic of Belarus \\ ${ }^{4}$ Belarusian State Medical University, Sukhaya Str. 28, Minsk, 220004, Republic of Belarus \\ ${ }^{5}$ Belarusian National Technical University, Nezavisimosty Ave. 65, Minsk, 220013, Republic of Belarus \\ ${ }^{1}$ Corresponding author \\ E-mail: 19lgimantas.bubulis@ktu.lt, ${ }^{2}$ rubnikovichs@mail.ru, ${ }^{3}$ ilya.khomich@gmail.com, \\ ${ }^{4}$ denisova_yul@mail.ru, ${ }^{5}$ vlad_minch@mail.ru
}

Received 20 June 2017; accepted 25 June 2017

DOI https://doi.org/10.21595/jme.2017.18797

Check for updates

Abstract. To study the effect of to low-intensity low frequency pulsed ultrasound (LILFPUS) on the surface wettability of titanium dental implants, an experimental medical-technical model was developed, consisting of an ultrasonic device, a fixed-mounted digital photo/video camera and a laboratory stand with a smooth vertical feed, in which an ultrasound nozzle with an experimental dental implant and fastener with a control implant were fixed. As a wetting agent, $0.9 \%$ sterile physiological sodium chloride solution was used in a petri dish tinted with brilliant green. Using the components of the model, it was possible to immerse the control and experimental dental implants, fixed on a laboratory stand, equally and uniformly at the same depth. The results of the studies showed that under the influence of ultrasound, the wetting of the surface of all the test samples was $100 \%$, and the wetting of the control samples was not observed.

Keywords: low intensity frequency, ultrasound, dental implantation, therapeutic device.

\section{Introduction}

An important direction in the treatment of patients with partial secondary adentia by dental implantation is to reduce the time from the installation of dental implants to their functional loading. One way to reduce the time of prosthetics for patients with partial secondary adentia is to optimize the processes of osseointegration of dental implants by changing the surface properties of implants and affecting the processes around them, starting from early postoperative periods. To do this, various chemical and physical methods are used to modify the surface of dental implants, including changes in geometry, wettability, micro- and nanotopography, aimed at improving molecular interactions and cellular response to implantation biomaterials to achieve more efficient osseointegration [1-7].

Among the many therapeutic measures used in the treatment of partial secondary adentia, the use of physical factors is of great importance. They are widely used at various stages of therapy, prevention and rehabilitation of dental diseases. The desire to achieve full osseointegration and reduce the complications rate after dental implantation encourages researchers to search for new, more effective therapies and their combinations [8-20].

Aim of the study. The aim of the study was to experimentally and clinically justify and prove the feasibility and effectiveness of using low-intensity pulsed ultrasound in dental implantation to help restore and form bone tissue around implants.

Material and methods. To evaluate the effect of low-intensity pulsed ultrasound on the wettability of the surface of titanium dental implants, 64 dental implants made of titanium Grade 5 were used in the experiment. The control group consisted of 32 dental implants without ultrasound exposure. The experimental group included 32 dental implants, which were exposed to low-intensity low frequency pulsed ultrasound (LILFPUS).

To study the nature of morphological changes in peri-implant bone tissues under the influence of the contact effect on the implants and peri-implant tissues with low-intensity pulsed ultrasound 
we performed experimental studies on 77 rabbits of the Chinchilla breed of both sexes, kept in steady conditions on a standard diet according to established standards in accordance with the rules for working with experimental animals. The animals were divided into three groups: the first group consisted of 27 rabbits with the traditional implantation method, the second group consisted of 25 rabbits with dental implants exposed to LILFPUS during implant insertion and the third group consisted of 25 rabbits with LILFPUS exposure of dental implants on insertion and subsequent LILFPUS exposure on the perimplant area (Fig. 1).

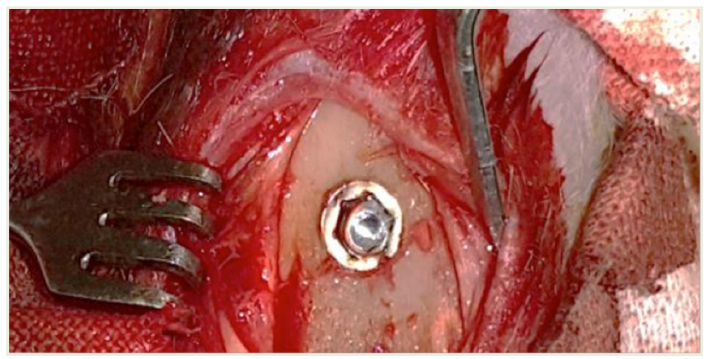

a)

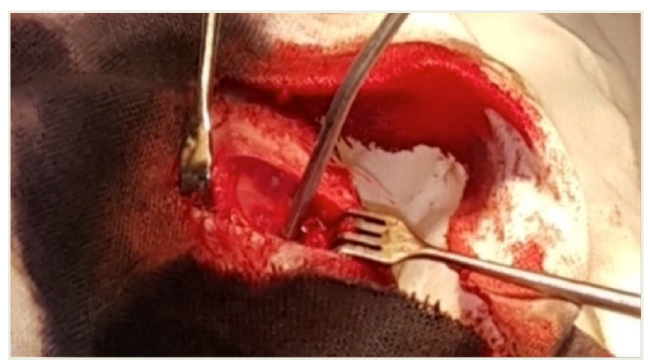

b)

Fig. 1. a) The installed dental implant, b) scoring the dental implant

At the end of the observation period, the animals were withdrawn from the experiment in accordance with the principles of bioethics (GLP standards). The study of micro-preparations and the production of micrographs were carried out with magnification of 100 and 400 using Axio Imager (Zeiss) and DMLS microscopes with software (Leica, Germany).

Morphometric analysis of histological preparations of bone samples was carried out at a magnification of 50 along the entire perimeter of each micropreparation using the Leica-Qwin software-hardware complex. The area of the active osteoblastic surface (So), the area of the newly formed (granulation, fibreotomic and bone) perimplant tissue (Sn) and the integration index (Io) were determined.

The clinical part was based on the results of a survey of 61 patients with a partial secondary adentia at the age of 35-44 years. Patients were divided into two groups, depending on the treatment used. The composition of the groups was uniform in severity of partial secondary adentia (Kennedy's third grade), age and sex of patients.

Patients of the first group had dental implantation carried out according to the traditional method, and in patients of the second group dental implantation was performed with a combined effect of low-intensity pulsed ultrasound on dental implants and perimplant tissues.

Statistical processing of data was carried out in accordance with modern requirements for conducting biomedical research using the application package Microsoft Excel 2010 (version 10.0, StatSoft, Inc., USA) and Statistica 6.0 software.

\section{Results of experimental studies on the wettability of the surface of dental implants}

To study the effect of LILFPUS on the surface wettability of titanium dental implants, an experimental medical-technical model was developed, consisting of an ultrasonic device, a fixedmounted digital photo/video camera and a laboratory stand with a smooth vertical feed, in which an ultrasound nozzle with an experimental dental implant and fastener with a control implant were fixed. As a wetting agent, $0.9 \%$ sterile physiological sodium chloride solution was used in a petri dish tinted with brilliant green.

Using the components of the model, it was possible to immerse the control and experimental dental implants, fixed on a laboratory stand, equally and uniformly at the same depth. Empirically, the base resonance frequency was determined for a given size of dental implants. During the immersion, test prototypes of dental implants were subjected to low-intensity low frequency 
pulsed ultrasound with the detected resonance frequency for 15 seconds.

The results of the studies showed that under the influence of ultrasound, the wetting of the surface of all the test samples was $100 \%$, and the wetting of the control samples was not observed. The time for complete wetting of the surface of the experimental dental implants subjected to low-intensity low frequency pulsed ultrasound was up to 12 seconds (Fig. 2).

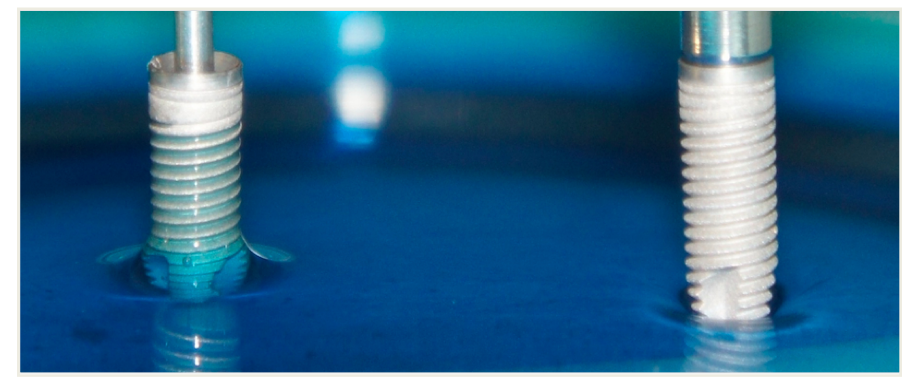

Fig. 2. Wetting of the surface of the dental implant under the influence of ultrasound (left)

Thus, the experiment proves the possibility to induce increased wettability effect on the surface of titanium dental implants under the influence of low-intensity low frequency pulsed ultrasound.

The results of morphological studies. After the installation of dental implants osteointegration processes in all groups of animals passed without disrupting the sequence of the repair stages. However, the timing and degree of reparation and osseointegration in the control and experimental groups of animals differed depending on the methods of treatment. In the early stages, granulation tissue was formed, which was later replaced by a fibrous reticular, coarse-fibrous and more mature lamellar bone tissue.

In the first group of rabbits, delayed maturation of bone tissue was noted, consisting of the presence of necrosis zones, immature granulation tissue, defects of the maternal bone in early periods, and predominance of coarse-fibrous bone tissue in later periods of osteosynthesis. Necrotic sites in the first group were noted at the early stages of the experiment. The processes of resorption in the first group of animals often prevailed over the processes of osteosynthesis.

The use of LILFPUS during dental implantation induces osteoreparation processes, stimulating neoangiogenesis in granulation and newly formed bone tissue. In 2 months in animals after implantation, the formation of bone tissue consisting of coarse fiber (predominant) with bone marrow in intertrabecular spaces and of lamellar bone, and in the peri-implantation area - a vascular network with actively functioning vessels of the microcirculatory bed.

Implantation in animals with a combined effect of low-intensity low frequency pulsed ultrasound on dental implants and peri-implant tissues of the operated region promoted the formation of bone tissue similar to the histostructure of the maternal bone. The trabeculae were oriented predominantly parallel to the implant surface, the osteocytes lay freely in the bone gaps, the bone plates were arranged in an orderly manner, thus preserving the histoarchitectonics of the bone tissue. In experimental groups of animals, a thickening of the periosteum was observed due to the proliferation of cells of the osteogenic layer or hyperplasia of the fibrous layer, as well as the invasion of the endostal and bone marrow blood vessels into the perimplant tissue.

The area of the newly formed perimplant tissue in groups of animals with the therapeutic effect of low-intensity pulsed ultrasound was significantly higher than in the group without LILFPUS at all times of the experiment. Complete integration of the newly formed tissue with the implant surface at all times of the experiment was observed in the third group of animals. In this group, a gradual replacement of the granulation and fibrous reticular tissue of the peri-implant area with a newly formed bone tissue, at first less mature coarse-fiber, and then mature lamellar, histoarchitectonically practically no different from the maternal bone.

The results of morphometric studies. As a result of the studies of the area of the active osteoblastic surface, it was established that, at the beginning and at the end of the experiment, the 
processes of osteoreparation were significantly higher in animals of the third experimental group than in the animals of the first group.

The results of statistical study of the area of the active osteoblastic surface showed that in the first group of animals where conventional treatment was used, the areas of bone trabeculae with a high density of osteogenic cells were significantly lower in all the periods of the experiment than in the third group (combined exposure to LILFPUS of implants and peri-implant tissues).

The area of the newly formed tissue along the perimeter of the implant bed was significantly higher in the animals of the second and third groups from the time of the initiation of the studies to the end of the observations, compared with those of the animals of the first group where traditional treatment was used.

Based on the studied indices of restoration of bone trabeculae area, as well as the complex of treatment, the highest results were obtained in animals of the third group. Thus, the combined effect of low-intensity pulsed ultrasound on implants and peri-implant tissues have a pronounced linear relationship - with combined use of low-intensity pulsed ultrasound, there is a pronounced increase in the area of the active osteoblastic surface.

After 1 and 2 months of the experiment, the formation of the newly formed tissue along the perimeter of the implant bed was observed in the second and third groups of animals. The formed tissue was represented by coarse-fiber and lamellar bone tissue. In the first group of rabbits, delayed maturation of bone tissue and the predominance of coarse-fibrous bone tissue in later periods of osteosynthesis were noted.

The obtained experimental results of the studies substantiate the advisability and advantages of the combined effect of low-intensity pulsed ultrasound on dental implants and peri-implant tissues for improving the processes of osteoreparation and osseointegration.

Description of the method of dental implantation with combined action of low-intensity pulsed ultrasound. During the insertion of dental implants, they were sonicated by low-intensity low frequency pulesd ultrasound in intermittent mode for 15 seconds, at a frequency of $32 \mathrm{kHz}$ using an ultrasonic device and a special waveguide concentrator that was tightly fixed in the implant to be installed (Fig. 3). Then, for 7 days, the peri-implant tissues of the operated jaw region, starting immediately after the operation, were subjected to contact action by low-intensity pulsed ultrasound at a frequency of $28 \mathrm{kHz}$ in intermittent mode using a labile technique for 10 minutes (Fig. 4).

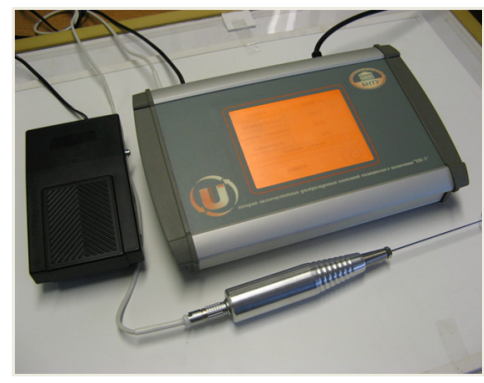

a)

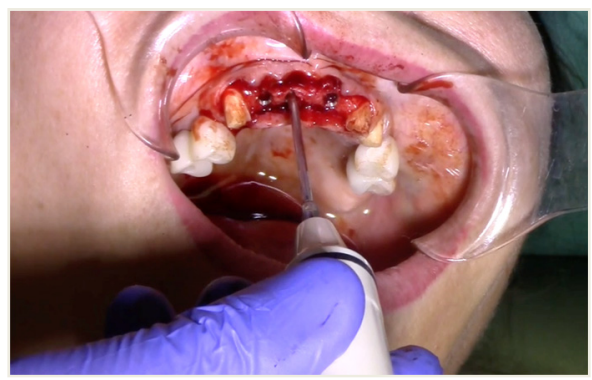

b)

Fig. 3. a) Ultrasonic device, b) sonication of implants

Comparative evaluation of the results of the standard and proposed method of dental implantation in patients with partial secondary adentia. In all patients before treatment, the reduced bone density of the jaw was determined according to cone-beam computer tomography. Indices of microcirculation of peri-implant tissues indicated changes of microcirculation in the form of a decrease in the intensity of microcirculation of peri-implant tissues.

According to the results of clinical observation of the first group of patients with partial secondary adentia in the first 8 days after dental implantation we traced the tendency to deterioration of all indicators in comparison with the initial state. At the same time, the mild degree 
of peri-implant tissues inflammation was maintained in form of pain of palpation, hyperemia, enlarged lymph nodes, edema of soft tissues of the cheek, restriction of opening of the mouth and deterioration of microcirculation despite good oral hygiene. The installation of dental implants led to a deterioration in the parameters characterizing inflammation and the state of microcirculation in peri-implant tissues.

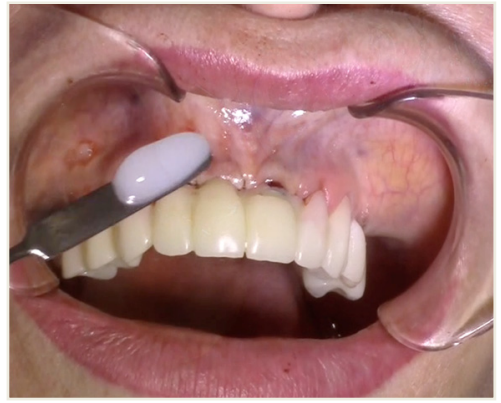

a)

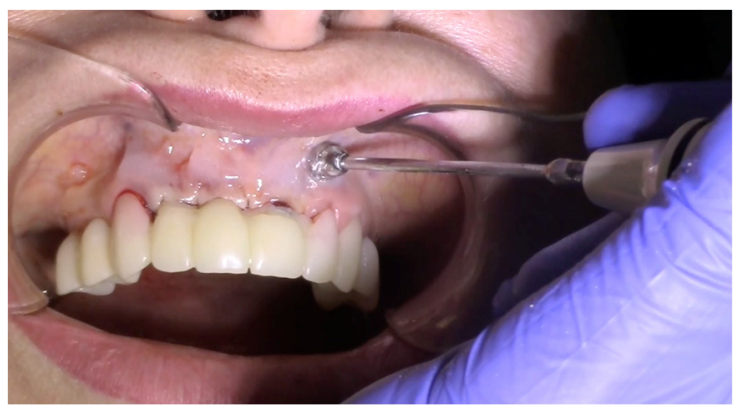

b)

Fig. 4. Contact ultrasound effect on the jaw tissue

During monitoring the second group of patients with partial secondary adentia, a positive effect of the combined effect of low-intensity pulsed ultrasound in dental implantation was established. Positive dynamics of decrease in intensity of inflammation in the nearest time of observation, starting from 3 days after exposure to LILFPUS was established, and by the 8th day of observation the decrease in inflammation was maximal.

There was a decrease in the period of acute postoperative condition (with a decrease in intensity or complete disappearance of pain), which is related to the analgesic and antiinflammatory effect of low-intensity pulsed ultrasound.

The use of ultrasound has a positive effect on the intensity of microcirculation in peri-implant tissues (by the end of the study, the microcirculation index in peri-implant tissues increased by $44.3 \%$ compared to the value before treatment).

The radiological picture after the installation of dental implants showed no active resorption processes and increased clarity of the pattern of spongy bone tissue. At the same time, in all patients of the second group, after only 2 months, the density of bone tissue of the alveolar process significantly increased in comparison with the first group. The increase in bone density at this observation period made it possible to conduct a second surgical stage of dental implantation, followed by prosthetics with non-removable cermeted structures supported by inserted dental implants. It was established that the complex treatment of the patients of the second group had a significant positive effect on bone density of the alveolar process in the area of the inserted implants, and by the end of the study the bone density index increased by $14.9 \%$ compared to the value before the treatment.

The use of low-intensity low frequency pulsed ultrasound facilitates the tolerability of dental implantation, contributes to the stable restoration of the normal physiological state of peri-implant tissues, helps to preserve the volume and improve the quality of the peri-implant bone.

Thus, the studies performed show that to improve the condition of peri-implant tissues in a complex of therapeutic and prophylactic measures in patients with partial secondary adentia, it is advisable to include LILFPUS during and after dental implantation.

\section{Conclusions}

Exposure to low-intensity pulsed ultrasound in experimental conditions allows to achieve $100 \%$ wetting of the surface of titanium dental implants [17]. Combined action of low-intensity pulsed ultrasound on dental implants and on peri-implant tissues of the operated region in animals 
promotes the formation of bone tissue approximating the histostructure to the maternal bone, with full and stronger integration of the newly formed bone tissue with the implant surface, which is manifested in more intensive bone formation (increase of osteoblastic surface compared with the control); more rapid maturation of granulation tissue and replacement of it with fibrous reticular in the early stages of reparative processes, the formation of trabecular and lamellar bone in later stages and the complete integration of the newly formed tissue with the implant surface [18]. The combined effect of low-intensity low frequency pulsed ultrasound in dental implantation is characterized by an increase in the density of the peri-implant bone tissue of the alveolar process, the weakening of inflammation, stimulation of the microcirculation of the mucosa of the peri-implant tissues, shortening of the acute postoperative condition, and of treatment time for restoring the integrity of the dentition, and consequently lowering the cost of treatment [19].

\section{References}

[1] Ivanov A. C. Fundamentals of Dental Implantology: Textbook. Allowance, 2013, (in Russian).

[2] Paraskevich V. L. Multiple immediate implantation. Synthesis of 15-year clinical experience. Dental Implantology and Surgery, Vol. 3, 2011, p. 80-100, (in Russian).

[3] Rubnikovich S. P. Treatment of patients with complete upper jaw adentia with removable prostheses based on dental implants. Dentist, Vol. 3, 2015, p. 29-36, (in Russian).

[4] Branemark P. I. Osseointegration and its experimental background. Journal of Prosthetic Dentistry, Vol. 50, Issue 3, 1983, p. 399-410.

[5] Le Guehennec L., et al. Surface treatments of titanium dental implants for rapid osseointegration. Dental Materials, Vol. 23, Issue 7, 2007, p. 844-854.

[6] Quaranta A., et al. A histomorphometric study of nanothickness and plasma-sprayed calciumphosphorous-coated implant surfaces in rabbit bone. Journal of Periodontology Online, Vol. 81, Issue 4, 2010, p. 556-561.

[7] Palmquist A., et al. Titanium oral implants: surface characteristics, interface biology and clinical outcome. Journal of The Royal Society Interface, Vol. 7, Issue 5, 2010, p. 515-527.

[8] Korchashkin N. B. Methods of Physiotherapy in Dental Implantology. 2002, p. 236, (in Russian).

[9] Musheev I. Practical dental implantology. Locus Standi, 2008, p. 497, (in Russian).

[10] Leung K. S., et al. Low intensity pulsed ultrasound stimulates osteogenic activity of human periosteal cells. Clinical Orthopaedics and Related Research, Vol. 418, 2004, p. 253-259.

[11] Khan Y. Fracture repair with ultrasound: clinical and cell-based evaluation. The Journal of Bone and Joint Surgery - American Volume, Vol. 90, Issue 1, 2008, p. 138-144.

[12] Ryzhkovskaya E. L., et al. The effect of low-frequency ultrasound on the joint capsule and the cartilage of the ankle in the experiment. Russian Medical Journal, Vol. 4, 2008, p. 64-66, (in Russian).

[13] Pounder N. M. Low intensity pulsed ultrasound for fracture healing: A review of the clinical evidence and the associated biological mechanism of action. Ultrasonics, Vol. 48, Issue 4, 2008, p. 330-338.

[14] Hasuike A., et al. In vivo bone regenerative effect of low-intensity pulsed ultrasound in rat calvarial defects. Oral Surgery, Oral Medicine, Oral Pathology, Oral Radiology, Vol. 111, Issue 1, 2011, p. $12-20$.

[15] Tobita K., et al. Effect of low-intensity pulsed ultrasound stimulation on callus remodelling in a gap-healing model: Evaluation by bone morphometry using three-dimensional quantitative micro-CT. The Journal of Bone and Joint Surgery, Vol. 93, Issue 4, 2011, p. 525-530.

[16] Bazylev N. B. Investigation of the stressed-strained state of cermet dentures using digital laser speckle-photographic analysis. Journal of Engineering Physics and Thermophysics, Vol. 82, Issue 4, 2009, p. 789-793.

[17] Denisova Y. L. Laser speckle technology in stomatology. Diagnostics of stresses and strains of hard biotissues and orthodontic and orthopedic structures. Journal of Engineering Physics and Thermophysics, Vol. 86, Issue 4, 2013, p. 940-951.

[18] Denisov L. A., Dedova L. N. Vacuum-d'arsonvalization in the treatment of parodontitis. Vopr Kurortol Fizioter Lech Fiz Kult, Vol. 2, 1982, p. 26, (in Russian).

[19] Dedova L. N., Denisov L. A. The treatment of apical periodontitis by using combined exposure to focal measured vacuum and local d'Arsonval treatment. Stomatologiia, Vol. 1, 1991, p. 26. 